\title{
Regulation of lactic acid concentration in its bioproduction from wheat flour
}

\author{
Karen Gonzalez ${ }^{(1),(2)}$, Sihem Tebbani*(2), Filipa Lopes ${ }^{(1)}$, \\ Aurore Thorigné( ${ }^{(3)}$, Sébastien Givry ${ }^{(3)}$, Didier Dumur ${ }^{(2)}$, Dominique Pareau ${ }^{(1)}$ \\ ${ }^{(1)}$ LGPM, CentraleSupélec, Université Paris-Saclay, Grande Voie des Vignes, 92295 Châtenay- \\ Malabry cedex, France \\ (2) Laboratoire des Signaux et Systèmes, CentraleSupélec, CNRS, Univ. Paris-Sud, Université \\ Paris-Saclay, Control Department, 3 Rue Joliot Curie, \\ 91192 Gif-Sur-Yvette cedex, France \\ ${ }^{(3)}$ Centre de Recherche et d'Innovation Soufflet-division Biotechnologie-Groupe SOUFFLET, \\ Quai Sarrail, 10400 Nogent-sur-Seine cedex, France \\ Corresponding Author*: Sihem Tebbani \\ e-mail: sihem.tebbani@ centralesupelec.fr \\ Phone number: $+33(0) 169851385$ \\ Fax: +33 (0)1 69851389
}

\begin{abstract}
Lactic acid is an important molecule for biopolymer production that can be obtained by biological processes. This work deals with the control of the lactic acid concentration in its production bioprocess using wheat flour as substrate. An adaptive control strategy for the simultaneous saccharification, proteins hydrolysis and fermentation (SSPHF) continuous process of lactic acid production is proposed in order to regulate the lactic acid concentration to the target value. The latter is determined so that the lactic acid productivity is maximized. The control strategy effectiveness and robustness are illustrated by means of experimental results.
\end{abstract}




\section{KEYWORDS}

Lactic acid production, kinetic-free adaptive control, feedback linearizing controller, wheat flour, production rate estimation.

\section{Introduction}

Due to the use of lactic acid as the monomer for the PLA (Poly Lactic Acid) production and the increasing need of manufacturing green plastics, lactic acid production has attracted a great interest recently. However, in contrast to petroleum-derived plastics, PLA production is still considered as an immature technology at the industrial scale. This is mainly due to the cost of the used raw material, lactic acid, which is highly dependent on the substrates and the fermentation process used (Abdel-Rahman et al., 2013). Employing alternative low cost substrates, improving and optimizing the fermentation process are therefore of utmost importance to reach a cost effective lactic acid production process. In this context, process control is required to improve the process production operation. The improvement of operational stability and production efficiency are the main goals when applying control methods to this type of biotechnological processes (Ben Youssef et al., 2005). Nevertheless, three main obstacles have hampered the development of modern control strategies in this field. First, since bioprocesses involve living organisms, their dynamics, strongly nonlinear and non-stationary, are often poorly understood; in addition, the replicability of experimental results is not guaranteed. Secondly, the microorganisms can be subjected to metabolic variations and physiological modifications over long operation periods, resulting in a change in the model parameters values over time. Finally, reliable sensors for real-time monitoring of key variables and control strategies implementation are often lacking (Bastin and Dochain, 1990). Prior modeling and online estimation become then necessary for the development of control strategies.

Despite these difficulties, several works in the literature on bioprocess control were reported during the last three decades. Proposed control techniques have been applied to various biotechnological processes such as biomass production, fermentation, anaerobic digestion, yeast and penicillin productions, microalgae cultures, etc. (Pons, 1991 ; Schubert et al., 1994 ; Roux et al., 1996 ; Hilgert et al., 2004 ; Mailleret et al., 2004 ; Marcos, et al., 2004 ; Ramaswamy et al., 2005 ; Jenzch et al., 2006 ; Selișteanu et al., 2007 ; Sbarciog et al., 2014).

In the case of lactic acid production, only few control strategies were proposed in the literature. Most of them concern fed-batch cultures (Choi, et al., 2014), while others deal with continuous cultures using glucose as substrate (Ben Youssef et al., 2000). An adaptive on-line optimizing control strategy for maximizing lactic acid productivity from glucose has been proposed in Shi et al. (1990). For continuous 
fermentation process, an adaptive predictive control strategy for regulating the biomass concentration was proposed by Dahhou et al. (1991). This predictive control scheme calculates the dilution rate from the online estimation of the specific growth rate (considered as a time varying parameter). The efficiency of the developed control strategy was evaluated by simulations. Moreover, a system with two bioreactors in cascade developed to maximize lactic acid production was developed in Ben Youssef et al.(2000) using glucose as substrate. The control approach regulated the substrate using an adaptive predictive control structure and online measurements of the substrate concentration. The specific growth and lactic acid production rates were estimated online using an asymptotic observer. This approach was not validated experimentally, but simulations were encouraging. Petre et al. (2011) further studied the previous system and proposed an indirect adaptive controller based on a dynamical neural network. The effectiveness of this control approach was proven by simulations.

Most of works presented previously consider glucose as substrate for fermentation. Nevertheless, the development of processes that use bioresources as substrate for biological conversions is of utmost importance. A control strategy for the fed-batch Simultaneous Saccharification and Fermentation process from Starch to Ethanol (SSFSE) was proposed in Ochoa et al. (2008). The goal of this control strategy was to maintain the glucose concentration at a quasi-steady state by feeding starch into the process. An adaptive approach was considered that estimates the glucose consumption and ethanol production rates in the bioreactor from starch and glucose concentrations, the latter being assumed to be measured. This approach was not experimentally validated due to the lack of online sensors to determine sugars concentrations.

The Simultaneous Saccharification and Fermentation process from starch was also studied in (Dai et al., 2014). The process was operated in batch mode and the effect of the temperature on the ethanol production was modeled by an energy-balance equation. The cooling rate and the enzymes addition were further optimized in simulation, leading to about $10 \%$ increase in the ethanol yield.

The literature survey clearly underlines that most of the works on lactic acid production do not validate experimentally their control strategies, although it is of prime importance when envisaging their further application in industrial facilities. This is one of the goals of this study. More specifically, the study presented hereafter will focus on the development of a control strategy for the SSPHF (Simultaneous Saccharification and Proteins Hydrolysis Fermentation) continuous process using wheat flour as the substrate for the process. The continuous mode was preferred to batch or fed-batch modes for two main reasons: (i) to avoid the inhibition effect of lactic acid on bacteria growth (Gonzalez et al. 2016), (ii) even if higher lactic acid concentrations are reached in batch or fed-batch fermentations, higher production rates are obtained in continuous fermentation where process shut down occurs less frequently (Hofvendahl et al. 
2000). The continuous mode is then more attractive for the industrial production of lactic acid. In the context of PLA production, this work is a pre-study for the optimization of the industrial production of lactic acid by bacteria from wheat flour using a dedicated control strategy. The proposed control law regulates the lactic acid concentration at a target value that maximizes its productivity using the feed flow rate as the control variable. It represents a first step in the development of an industrial process of lactic acid production.

The article is organized as follows. The next section describes the experimental set-up followed by the system modelling. Then, the control design is presented. A feedback linearizing control approach is considered in the first place and then modified in order to reduce the control law complexity and increase its robustness with respect to model uncertainties. An adaptive control law is then proposed using the lactic acid production rate estimation. The adaptive controller is validated experimentally and its robustness regarding operational factors disturbances is evaluated. Finally, concluding remarks and perspectives are stated at the end. In Appendix, the convergence of the estimation and control strategies are analyzed.

\section{Materials and Methods}

\subsection{Microorganism and culture conditions}

Lactobacillus coryniformis subsp. torquens DSM 20004 is stored at $-80^{\circ} \mathrm{C}$ in Lactobacilli MRS medium with $40 \%$ glycerol. Pre-cultures were prepared by proliferation of a stock culture to $100 \mathrm{~mL}$ MRS medium and cultured in an incubator shaker MAXQ 4000 (Thermo Scientific) at $30^{\circ} \mathrm{C}$ for $12 \mathrm{~h}$. A second proliferation was done at $30^{\circ} \mathrm{C}$ in $1000 \mathrm{~mL}$ culture medium during $12 \mathrm{~h}$. The cells were then harvested after centrifugation $\left(3000 \mathrm{~g}, 3 \mathrm{~min}, 20^{\circ} \mathrm{C}\right)$, resuspended in $100 \mathrm{~mL}$ distilled water. This suspension was then used for the fermenter inoculation (corresponding to $3 \%$ of the total working volume of the fermenter).

\subsection{Bioreactor description}

The studied set-up consists of a continuous stirred tank reactor (CSTR) (Global Process Concept, La Rochelle France) illustrated in Figure 1. Five variables are controlled: temperature, $\mathrm{pH}$, culture broth level in the bioreactor, agitation and feed flow rate. The temperature, $\mathrm{pH}$ and broth level are controlled by PID controllers using temperature, $\mathrm{pH}$ and foam sensors. 
A mechanical overhead stirring device ensures the medium mixing and is regulated by a PID controller which adjusts the motor speed of the agitator to a setpoint value. Finally the feed flow rate is regulated by a peristaltic pump with variable rotation speed.

\subsection{Bioprocess description}

The whole process studied in this work is divided into three steps. First, in the liquefaction step (see below), starch is liquefied from wheat flour into maltose and glucose. In a second step, the simultaneous saccharification and wheat proteins hydrolysis (SSPH) is performed to partially hydrolyze maltose and wheat proteins into glucose and amino acids, respectively. This step is performed in order to make carbon and nitrogen sources available to the bacteria. In the final step, a simultaneous saccharification proteins hydrolysis and fermentation (SSPHF) allows hydrolyzing the remaining maltose and wheat proteins simultaneously with the fermentation. The liquefaction and SSPH steps are performed in batch mode. This paper will focus on the SSPHF process. Two types of bioreactors are used: a 5 L bioreactor (Fig. 1) where the continuous SSPHF step takes place, and a 12 L bioreactor (Fig. 2) which contains the partially hydrolyzed wheat flour solution that feeds the $5 \mathrm{~L}$ bioreactor. Liquefaction and SSPH steps are performed in both reactors. SSPHF step is only performed in the $5 \mathrm{~L}$ reactor.

\subsection{Liquefaction}

The whole wheat flour was suspended in water at a concentration of $260 \mathrm{~g} \mathrm{~L}^{-1}$, heated to $50^{\circ} \mathrm{C}$ and agitated at $400 \mathrm{rpm}$ in a $5 \mathrm{~L}$ bioreactor. The $\mathrm{pH}$ was regulated at 5.5, with addition of sodium hydroxide and sulfuric acid. The liquefaction of wheat flour was performed using two enzymes: Lyvanol devisco and Lyquozyme SDCS (Lyven, Colombelles, France), each at a concentration of $94 \mu \mathrm{L} \mathrm{L}^{-1}$. After enzymatic reaction, the mixture was heated to $85{ }^{\circ} \mathrm{C}$ and maintained at this temperature for about $30 \mathrm{~min}$. Finally, the temperature was reduced to $50{ }^{\circ} \mathrm{C}$ for the SSPH step.

\subsection{Simultaneous saccharification and proteins hydrolysis (SSPH)}

For the SSPH step, the $\mathrm{pH}$ was adjusted to 5.7 , the temperature regulated at $50^{\circ} \mathrm{C}$ and the agitation in the bioreactor set to $150 \mathrm{rpm}$. Two enzymes were added: Maltose saccharification was performed by the Amyloglucosidase enzyme (AMG) (Lyvanol GA, Lyven, Colombelles, France), at a concentration of 230 $\mu \mathrm{L} \mathrm{L}^{-1}$. Protein hydrolysis was performed by the Prolyve NP enzyme (Lyven, Colombelles, France) at a concentration of $560 \mu \mathrm{L} \mathrm{L}^{-1}$. These conditions were maintained during $6 \mathrm{~h}$, and then, the temperature was decreased to $30{ }^{\circ} \mathrm{C}$ for the SSPHF step. 


\subsection{Hydrolyzed wheat stock}

The wheat solution at $260 \mathrm{~g} \mathrm{~L}^{-1}$ was liquefied, and then hydrolyzed in a $12 \mathrm{~L}$ bioreactor with the conditions described previously (sections 2.4 and 2.5) in order to produce the stock solution necessary to feed continuously the SSPHF bioreactor. The temperature was maintained at $12{ }^{\circ} \mathrm{C}$ in order to stop the enzymatic activity.

\subsection{Simultaneous saccharification, proteins hydrolysis and fermentation (SSPHF)}

The SSPHF step started immediately after inoculation. The $\mathrm{pH}$ was measured continuously, and kept constant at 5.7 with addition of $7 \mathrm{M}$ sodium hydroxide. The temperature was kept at $30^{\circ} \mathrm{C}$ and the stirring rate in the reactor was maintained at $150 \mathrm{rpm}$.

\subsection{Analyses}

Samples were withdrawn from the SSPHF bioreactor at various time intervals in order to measure cell, substrates (maltose and glucose) and lactic acid concentrations offline. Cell concentration was measured by cell counting in a Thoma cell counting chamber. A good linear correlation between biomass

concentration $X$, in $10^{10}$ cells $\mathrm{L}^{-1}$, and dry weight $X_{\mathrm{dry}}$ (Total suspended solids), in $\mathrm{g} \mathrm{L}^{-1}$, was obtained from experimental assays: $X_{\mathrm{dry}}=0.70 \mathrm{X}$.

Concentrations of glucose, maltose and lactic acid were determined by HPLC (Waters Alliance HPLC system, Water Corporation, Milford, MA) employing a Shodex Sugar column (Shodex, Japan. $45{ }^{\circ} \mathrm{C}$ with

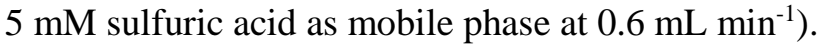

Standard deviations of biomass measurements (cell counting) were calculated according to Niemelä and Keskus (2002). Standard deviations of maltose, glucose and lactic acid concentrations were determined considering uncertainties related to the HPLC method and to sampling. The determined measurement errors were about $10 \%$ of the measured values.

\subsection{Control law implementation}

The C-BIO software (Global Process Concept, La Rochelle France) was used for the implementation of the control law. The sampling time of the software was set to 5 minutes. The control objective was to regulate the lactic acid concentration to its optimal value. The adaptive control strategy was then experimentally evaluated to assess its performance (step variations of the set point) and robustness (temperature disturbance). Only constant or ascending changes in the reference values were considered. 
The control performance was evaluated comparing the online calculation of lactic acid concentration (see section 3.2) with its offline measurements.

\section{Modelling and Control Design}

\subsection{Process modelling}

Mechanisms involved in maltose saccharification and lactic bacteria metabolism must be considered when establishing the mathematical model of the bioprocess: biomass growth, substrate (glucose) consumption, product (lactic acid) formation and maltose degradation. Glucose is assumed as the limiting substrate in the system (Gonzalez et al., 2016). Dynamics of these variables are based on mass balance equations for a continuous stirred tank reactor. Residence time distribution experiments (data not shown) showed that the whole volume of the bioreactor is not perfectly stirred, with $91 \%$ of effective volume. This was taken into account for the modeling and in the control design when determining the dilution rate, $D$ (the ratio of the feed flow rate over the effective reactor volume, in $\mathrm{h}^{-1}$ ). Dynamics of cell, glucose, lactic acid and maltose concentrations are described by the following equations (Gonzalez et al., 2016):

$$
\begin{aligned}
& \frac{d X}{d t}=\mu X-D X \\
& \frac{d S}{d t}=-\frac{1}{Y_{X S}} \mu X+K_{M} M+D\left(S_{0}-S\right) \\
& \frac{d P}{d t}=\frac{Y_{P S}}{Y_{X S}} \mu X-D P \\
& \frac{d M}{d t}=-K_{M} M+D\left(M_{0}-M\right)
\end{aligned}
$$

where $X, S, P, M$ are the cell, glucose, lactic acid and maltose concentrations (in $\mathrm{g} \mathrm{L}^{-1}$ ), respectively. $\mu$ is the specific growth rate (in $\left.\mathrm{h}^{-1}\right), Y_{P S}$ and $Y_{X S}\left(\right.$ in $\mathrm{g} \mathrm{g}^{-1}$ ) are the product and biomass yields with respect to glucose, respectively. $K_{M}$ is the maltose degradation constant (in $\left.\mathrm{h}^{-1}\right) . M_{0}\left(50 \mathrm{~g} \mathrm{~L}^{-1}\right)$ and $S_{0}\left(120 \mathrm{~g} \mathrm{~L}^{-1}\right)$ represent the maltose and glucose concentrations feeding the SSPHF bioreactor, respectively.

The model describing the bacteria growth kinetics as well as the parameters in equations (1) to (4) was determined in a previous study (Gonzalez et al., 2016). The specific growth rate is expressed by the Levenspiel generalization of the Monod equation (Levenspiel 1980): 
$\mu=\mu_{\max } \frac{S}{K_{S}+S}\left(1-\frac{P}{P_{\max }}\right)^{n}$

where $\mu_{\max }$ is the maximal specific growth rate (in $\mathrm{h}^{-1}$ ), $K_{S}$ the half saturation constant, $n$ the inhibiting power concerning lactic acid and $P_{\max }$ the maximal lactic acid concentration (in $\mathrm{g} \mathrm{L}^{-1}$ ).

Values of the model parameters were identified from experimental data (Gonzalez et al., 2016) and are given in Table 1.

\subsection{Online determination of lactic acid concentration}

All the compounds concentrations are measured offline. For control purposes, at least one online measurement is needed. In this study, the added sodium hydroxide amount used to regulate the $\mathrm{pH}$ in the bulk is the sole measurement available online. Hereafter, the $\mathrm{pH}$ variation is assumed to be mainly due to lactic acid production. A small amount of sodium hydroxide is consumed to neutralize the produced amino acids; in most cases it is negligible compared to sodium hydroxide consumed for lactic acid neutralization. Thus, sodium hydroxide (a strong base) will neutralize lactic acid (a weak acid): the molar amount of lactic acid produced equals the one of sodium hydroxide added (one mole of sodium hydroxide reacts with one mole of lactic acid). Consequently, the evolution of the lactic acid concentration can be expressed with respect to the sodium hydroxide concentration using the following expression (expressed in concentrations in $\mathrm{g} / \mathrm{L}$ ):

$V \dot{P}+F \quad P=F_{N a} C_{N a}$

where $F$ and $F_{N a}$ are the medium and sodium hydroxide inlet flow rates respectively (in $\mathrm{L} \mathrm{h}^{-1}$ ), $V$ is the effective volume of the bioreactor (in L), $C_{N a}$ is the sodium hydroxide concentration (in $\mathrm{g} \mathrm{L}^{-1}$ ) in its inlet flow $\left(F_{N a}\right)$. In equation (6), the total quantity of lactic acid molecules produced per unit time, defined as the sum of accumulated molecules in the reactor $(V \dot{P})$ and those leaving the system $(F P)$, is equal to the added quantity of sodium hydroxide per unit time $\left(F_{\mathrm{Na}} C_{\mathrm{Na}}\right)$.

From (6), the lactic acid concentration can be determined online using the following discretized equation:

$P_{k+1}=\left(F_{N a, k} \frac{C_{N a}}{V}-D_{k} P_{k}\right) T_{s}+P_{k}$ 
where $k$ in subscript represents the discrete time index and $T_{s}$ the time interval duration. Lactic acid concentration at instant $k+1$ is thus calculated from the inlet sodium hydroxide flow rate, the dilution rate and the lactic acid concentration at instant $k$. The relation (7) was validated through experimental data (data not shown).

\subsection{Control design}

The range of optimal operation conditions to maximize lactic acid productivity was determined in a previous study (Gonzalez et al., 2016). The aim here is to maintain the process close to these optimal conditions (lactic acid production rates must be regulated at values comprised between 2.8 and $2.9 \mathrm{~g} \mathrm{~L}^{-1} \mathrm{~h}^{-1}$ corresponding to lactic acid concentrations between 18 and $25 \mathrm{~g} \mathrm{~L}^{-1}$ ). A control law is thus designed to regulate the lactic acid concentration to a given setpoint within this optimal range of values. The objective is to propose a control scheme that presents the best trade-off between performance, robustness, simplicity of implementation, cost efficiency (requiring non expensive sensors) in the perspective of future work with an industrial bioreactor. Hereafter, the proposed control structure, that ensures a good compromise between the previously mentioned criteria, is detailed.

The system equations (1) to (4) can be rewritten in the state-space formalism as a single-input singleoutput model:

$$
\begin{aligned}
\dot{\mathbf{x}}(t) & =\mathbf{f}(\mathbf{x}(t))+\mathbf{g}(\mathbf{x}(t)) u(t) \\
y(t) & =h(\mathbf{x}(t))
\end{aligned}
$$

where $\mathbf{x}=(X, S, P, M)^{T}, u=D$ and $y=P$. Note that the control input $u$ appears linearly in equation (8), thus, this system is a control-affine one.

In conventional linear control strategies of nonlinear plants, one first calculates a linearized approximation of the model (8) around an equilibrium point, and then the control design is achieved using a linear controller based on the approximate model (e.g. PID controller). However, the closed-loop remains nonlinear and its global stability is difficult to assess, and could possibly be only valid around the linearizing point.

For nonlinear control-affine systems (as the system studied here), one possibility is to use the state feedback linearizing control law (Isidori, 1989). In this approach, widely used in bioprocess control, the nonlinear system is fully or partially linearized, and linear control techniques are further applied to the linearized model. The feedback linearization control is in general more efficient than the first-order Taylor based linearization approach because it is achieved by exact state transformation and feedback, rather than 
by linear approximations of the dynamics. However, this control approach is model-based and consequently, its performance highly depends on the model accuracy. In addition, as it will be presented hereafter, this control strategy involves analytical derivation of the dynamics with respect to the state variables. Thus, its application to complex or black-box models could be very difficult, even impossible.

The proposed controller structure, illustrated in Figure 3, consists of two loops: an input-output linearizing controller in an inner loop, with a proportional controller in the outer loop. A Proportional Integral controller could be also designed in the outer loop to ensure a good reference tracking and to reject disturbances. However, an anti-windup mechanism must be included in this case since the control input is subject to saturation (Tebbani et al., 2015).

The linearizing controller aims at cancelling the nonlinearities in the system dynamics. It will transform the nonlinear plant into an equivalent linear one, through a state transformation and a nonlinear state feedback.

In order to design the input-output linearizing controller, the relative degree of system (8) has to be determined (Isidori, 1989). The relative degree, denoted $r$ (with $r \leq n=\operatorname{dim}(\mathbf{x})$ ), is the lowest order time derivative of the output $y$ that is directly related to the control input $u$. If the system has a relative degree $r=n$, then it can be totally linearized by means of an input-output feedback and will be equivalent to $n$ integrators. Otherwise, it can be partially linearized and its input-output behavior will be identical to $r$ integrators.

This equivalent linear model has a new input $\hat{u}$ (derived from the outer loop as the output of the outer controller, following a cascaded scheme). The state feedback $u$ is given by:

$u=\frac{\hat{u}-L_{\mathrm{f}}^{r} h(\mathbf{x})}{L_{\mathrm{g}} L_{\mathrm{f}}^{r-1} h(\mathbf{x})}$

where $L_{\mathrm{f}} h$ is the Lie derivative of $h$ in the direction $\mathbf{f}$, with $L_{\mathrm{f}} h(\mathbf{x})=\frac{\partial h}{\partial \mathbf{x}} \mathbf{f}(\mathbf{x}), L_{\mathrm{f}}^{r} h(\mathbf{x})=\frac{\partial h}{\partial \mathbf{x}} L_{\mathrm{f}}^{r-1} h(\mathbf{x})$ and $L_{\mathrm{g}} h(\mathbf{x})=\frac{\partial h}{\partial \mathbf{x}} \mathbf{g}(\mathbf{x})$

The input $\hat{u}$ is determined so that the closed-loop plant achieves the desired performance. The outer controller is a linear controller, chosen depending on the relative degree of the plant and on the aimed closed-loop performance. 
Since the aim of the control law is to regulate the product concentration, formulae (9) is applied to model (4), leading to the following control law (here $r=1$ ):

$D=\frac{1}{P}\left[\frac{Y_{P S}}{Y_{X S}} \mu X-\hat{D}\right]$

In this work, the control signal $\hat{D}$ in equation (10) is derived from a Proportional controller by the following equation:

$\hat{D}=G\left(P_{r e f}-P\right)$

where $P_{r e f}$ is the reference lactic acid concentration and $G$ the proportional controller gain, tuned to provide a desired closed-loop time response. Indeed, the inner-loop is equivalent to an integrator (since the relative degree $r$ equals 1) and thus the closed-loop system with the Proportional controller is equivalent to a first-order system with a time constant equal to $1 / G$. The gain $G$ is then directly derived from the desired time response of the closed-loop system. At initial time, if $P(0)=0$, the control input is first set to $D=0$ instead of using (10). Afterwards, since the product concentration increases, the formulae (10) can be used.

The control law given by equations (10)-(11) presents two main drawbacks. First, it needs the online knowledge of all state variables $(X, S$ and $P$ ). However, in the considered system, only the lactic acid concentration is available (see section 3.2). Thus, estimators are needed in order to reconstruct $X$ and $S$ values leading to a complex control system. Indeed, since the system model is nonlinear and uncertain, estimators for this type of system are difficult to develop and implement (see e.g. (Gonzalez et al., 2015b) for the implementation of an Unscented Kalman Filter coupled to the linearizing controller). Secondly, the control law in equation (10) involves the knowledge of the specific growth rate $\mu$. Consequently, its performance highly depends on the accuracy of the growth kinetic.

In order to simplify the control law structure and to increase its robustness with respect to model uncertainties, a kinetic-free adaptive control law is further proposed hereafter. It consists in estimating, online, the lactic acid production rate, denoted $\gamma$ and defined as follows:

$\gamma=\frac{Y_{P S}}{Y_{X S}} \mu X$

By introducing $\gamma$ in equation (10) and replacing $\gamma$ by its estimate $\hat{\gamma}$, the control law given by equation (10) becomes: 
$D=\frac{1}{\hat{P}}[\hat{\gamma}-\hat{D}]$

where $\hat{D}$ is the linear control input and is still given by (11) (by replacing $P$ with $\hat{P}$ ), and $\hat{\gamma}$ is the estimated production rate. The control law needs the knowledge of product concentration $\hat{P}$. The latter could be either the calculated product concentration from the measurement of sodium hydroxide added, or its estimated value (see section 3.4).

The advantages of this controller are the following: (i) the estimation algorithm is simplified in comparison to the estimation of the state variables, and (ii) the controller (13) does not involve any growth model, leading to a more robust control strategy than the feedback linearizing control law in equation (10). As $D$ in equation (13) can be negative or of very high values that are not physically feasible, this variable is further bounded in the interval $\left[0, D_{\max }\right]$ where $D_{\max }$ is the maximal dilution rate allowed by the pumps, here equal to $0.31 \mathrm{~h}^{-1}$.

The control input given by (13) is applied after saturation to the plant via a $\mathrm{ZOH}$ device, i.e., it is maintained constant between two sampling instants.

The structure of the implemented control law, that includes the production rate estimation, is illustrated in Fig. 4. In this case the product concentration used in the control law (13) is the calculated one from the measurement of added sodium hydroxide. This choice will be discussed in section 4 .

The stability of the proposed control law is analysed in Appendix A.3, under assumptions presented in Appendix A.1 and based on estimation strategy convergence (analysed in Appendix A.2).

\subsection{Production rate estimation}

The control law given by equation (13) involves the estimation of the production rate. The latter is assumed to be constant between two sampling instants. In order to validate the proposed approach, a study, in simulation, was carried out to compare several estimation approaches for this variable; namely numerical differentiation, linear model and constant model (Gonzalez et al., 2015a). A linear model for the production rate dynamics was shown to present the best trade-off between performance and simplicity of implementation. It was then retained for the experimental validation and is detailed hereafter.

The model of the system to be considered for the estimation problem is described as follows:

$$
\begin{aligned}
& \left\{\begin{array}{l}
\dot{P}=\gamma-D P \\
\dot{\gamma}=0
\end{array}\right. \\
& y=P
\end{aligned}
$$


where $\dot{P}$ represents the first time derivative of $P$, and $y$ is the output.

First, this model is time-discretized. Indeed, the control and estimation strategies will be implemented online in a discrete form. Since the system dynamics are slow enough (about hours) in comparison to the chosen sampling time ( $\left.T_{s}=5 \mathrm{~min}\right)$, the Euler discretization scheme provides good approximation. With a sampling time $T_{s}$, (14) is approached by a discrete-time model:

$$
\begin{aligned}
& \left(\begin{array}{l}
P_{k+1} \\
\gamma_{k+1}
\end{array}\right)=\left(\begin{array}{cc}
-D_{k} T_{s}+1 & T_{s} \\
0 & 1
\end{array}\right)\left(\begin{array}{l}
P_{k} \\
\gamma_{k}
\end{array}\right) \\
& y_{k}=P_{k}
\end{aligned}
$$

The objective here is to design an estimation algorithm based on model (15) for system (1)-(4). A Kalman filter is retained in this work (Lewis et al., 2008).

The model and measurement errors are modelled as additive noises. The stochastic time-discretized model is then given by:

$$
\begin{aligned}
& \left(\begin{array}{l}
P_{k+1} \\
\gamma_{k+1}
\end{array}\right)=\left(\begin{array}{cc}
-D_{k} T_{s}+1 & T_{s} \\
0 & 1
\end{array}\right)\left(\begin{array}{l}
P_{k} \\
\gamma_{k}
\end{array}\right)+\left(\begin{array}{l}
v_{1, k} \\
v_{2, k}
\end{array}\right) \\
& P_{k}=\left(\begin{array}{ll}
1 & 0
\end{array}\right)\left(\begin{array}{l}
P_{k} \\
\gamma_{k}
\end{array}\right)+w_{k}
\end{aligned}
$$

where $v_{1,2}$ and $w$ are the process and measurement noises, respectively. They are assumed to be non correlated Gaussian white noises as per the Kalman filtering theory (Lewis et al., 2008). Their covariance matrices are denoted $\mathbf{Q}$ and $\mathbf{R}$ respectively (here $\mathbf{R}$ is a scalar). They are chosen based on the knowledge on the model and measurement uncertainties. In this work, they are empirically chosen (by trial/error) in order to ensure an acceptable rate of convergence and the stability of the filter in simulation. Then, the best values were used for the experimental validation. The covariance matrix of the estimation error, denoted hereafter $\boldsymbol{\Sigma}$, is initialized according to the uncertainty on the determination of the lactic acid concentration and of the production rate at initial time. Off-line measurements of lactic acid concentration can be used to evaluate this uncertainty.

In this work, model (16) is treated as a Linear Time-Varying (LTV) model. It is rewritten as follows:

$$
\begin{aligned}
& \mathbf{x}_{k+1}=\mathbf{A}_{k} \mathbf{x}_{k}+\mathbf{v}_{k} \\
& y_{k}=\mathbf{C} \mathbf{x}_{k}+w_{k} \\
& \text { with } \mathbf{A}_{k}=\left(\begin{array}{cc}
-D_{k} T_{S}+1 & T_{S} \\
0 & 1
\end{array}\right), \mathbf{C}=\left(\begin{array}{ll}
1 & 0
\end{array}\right), \mathbf{v}_{k}=\left(\begin{array}{ll}
v_{1, k} & v_{2, k}
\end{array}\right)^{\mathrm{T}}, \mathbf{x}_{k}=\left(\begin{array}{ll}
P_{k} & \gamma_{k}
\end{array}\right)^{\mathrm{T}}, y_{k}=P_{k} \text {. }
\end{aligned}
$$


A linear discrete-time Kalman filter can be applied to system (17) to estimate the state variables from the available measurements (Lewis et al., 2008).

The convergence of the estimation strategy is analysed in Appendix A.2 considering assumptions presented in Appendix A.1.

Remark 1. The model (16) could be also treated as a nonlinear model, and an extended Kalman filter (EKF) could be used (Lewis et al., 2008). Since the model is bilinear with respect to the state variables and the control input, the derived equations from the EKF are identical to those derived while treating the model as an LTV model.

\section{Results and discussion}

The adaptive control law was experimentally validated in two cases: for a setpoint tracking and for the rejection of a disturbance. Obtained results are presented and discussed hereafter. The proportional controller gain $G$ in (11) was set equal to $6 \mathrm{~h}^{-1}$ which corresponds to a time response of about $30 \mathrm{~min}$. The covariance matrices $\mathbf{Q}$ and $\mathbf{R}$ for the Kalman filter are chosen diagonal as follows: $\mathbf{Q}=\operatorname{diag}([0.01 ; 0.01])$, $\mathbf{R}=0.01$. The estimate state at time $\mathrm{t}=0$ is set to $(\hat{P}(0) \quad \hat{\gamma}(0))=\left(\begin{array}{ll}10^{-6} & 0\end{array}\right)$. All the assumptions made in Appendix A.1 are fulfilled for the chosen design parameters. Error bars on the measured concentrations were determined as detailed in section 2.8 .

The performance in the case of a setpoint tracking and a change in the setpoint is first evaluated (Fig. 5). The setpoint was set to $P_{r e f}=20 \mathrm{~g} \mathrm{~L}^{-1}$ whereas the lactic acid concentration at initial time is $P(0)=0 \mathrm{~g} \mathrm{~L}^{-1}$. The control is activated after inoculation (addition of bacteria to the fermenter). First, the control law sets the bioreactor to operate in batch mode (i.e. $D=0$ ) to increase the lactic acid concentration. In this case, the system is in open-loop and the dynamics is imposed by the microorganisms' growth rate. When the setpoint is reached, after 10 hours, the reactor starts to operate in continuous mode (i.e. in closed-loop); the dilution rate is increased in order to maintain the lactic acid concentration constant and equal to $20 \mathrm{~g} \mathrm{~L}^{-}$ 1. Afterwards, the setpoint was changed from $20 \mathrm{~g} \mathrm{~L}^{-1}$ to $27 \mathrm{~g} \mathrm{~L}^{-1}$ at time $t=26 \mathrm{~h}$. The reactor operates therefore in batch mode in order to increase lactic acid concentration. Once it reaches the new setpoint, the dilution rate is increased. This time, the dilution rates calculated by the control law are lower than those determined during the first phase with a set point of $20 \mathrm{~g} \mathrm{~L}^{-1}$. The reference value was reached with good transient behavior and without overshoot. The calculated value of lactic acid concentration lies within the

confidence interval of its measurements. It can be noticed that the noise on lactic acid concentration (very small noise) leads to fluctuations on the applied dilution rate. Indeed, since the calculated product concentration was used in the control law instead of the estimated one, the measument noise on sodium 
hydroxide measurements is not filtered, leading to fluctuations on the control input. Nevertheless, this does not affect the control law performance. The estimated product concentration was not used here to avoid to use mismatched data at the beginning of the culture (in the transient time of the Kalman filter). However, since in the beginning of the culture, the system operates in batch mode, it is possible to use the estimated value without loss of performance (the error on the value of the product concentration will not affect the control input in this mode). The Kalman filter converges in less than 1 hour (from a simulation study) and in this case the measurement noise will be filtered. This choice will be tested in a future work.

In addition, in this experiment the production rate is around $2.7 \mathrm{~g} \mathrm{~L}^{-1} \mathrm{~h}^{-1}$ in the first phase (setpoint $=20 \mathrm{~g}$ $\mathrm{L}^{-1}$ ) and slightly decreases to $2.1 \mathrm{~g} \mathrm{~L}^{-1} \mathrm{~h}^{-1}$ (setpoint $=27 \mathrm{~g} \mathrm{~L}^{-1}$ ) afterwards. This is in agreement with results presented in (Gonzalez et al., 2016) and is similar to the one reported in (Ben Youssef et al., 2000) obtained with an adaptive predictive control law in simulation.

Concerning the state variables behaviour, cell concentration, being directly linked to lactic acid production, increased during the first batch phase and remained constant during the continuous phases. The maltose concentration decreased in the batch phases proving its enzymatic conversion to glucose. The glucose concentration is slightly affected, it remained constant during the whole experiment as a result of its continuous supply in the feed flow, its production by enzymatic activity and its bacterial consumption.

In a second step, the robustness of the adaptive control law with respect to disturbances was also studied by applying a temperature disturbance to the system. In this experiment, at $t=17 \mathrm{~h}$, the temperature was reduced from $30^{\circ} \mathrm{C}$ to $20^{\circ} \mathrm{C}$ until the end of the experiment (Fig. 6). A significant reduction in lactic acid production rate is observed (from $2.1 \mathrm{~g} \mathrm{~L}^{-1} \mathrm{~h}^{-1}$ to $1.1 \mathrm{~g} \mathrm{~L}^{-1} \mathrm{~h}^{-1}$ ). This is in agreement with data obtained in batch mode (data not shown), where lactic acid concentrations obtained at $30^{\circ} \mathrm{C}$ was 4 times higher than those obtained at $20^{\circ} \mathrm{C}$ with production rates of $1.5 \mathrm{~g} \mathrm{~L}^{-1} \mathrm{~h}^{-1}$ and $0.4 \mathrm{~g} \mathrm{~L}^{-1} \mathrm{~h}^{-1}$, respectively.

Moreover, the productivities obtained in the batch and continuous modes at $30^{\circ} \mathrm{C}$ and for $P=20 \mathrm{~g} \mathrm{~L}^{-1}$ and were calculated and found about $1.38 \mathrm{~g} \mathrm{~L}^{-1} \mathrm{~h}^{-1}$ and $2.7 \mathrm{~g} \mathrm{~L}^{-1} \mathrm{~h}^{-1}$ in batch and in continuous modes, respectively. The productivity obtained in the continuous mode is almost twofold higher than the one determined in the batch one. This proves the advantage of operating the bioprocess in continuous mode over the batch operation.

Despite the disturbance, the developed control law regulates and stabilizes the lactic acid concentration at its reference value by reducing the dilution rate, which confirms its robustness with respect to this kind of disturbance. Lactic acid concentration remains at it reference value with a maximal absolute error of $0.7 \mathrm{~g} \mathrm{~L}^{-1}$ (corresponding to $2.6 \%$ ). It is therefore not affected by the disturbance and the dilution rate 
quickly stabilizes. It is worth noting that no integral action was added to reject this disturbance. Indeed, since the controller uses directly the estimate of the production rate, it can reject any disturbances more efficiently than a classical, model-based, state feedback linearizing control law. Concerning the behaviour of the state variables, biomass, glucose and maltose concentrations remained constant during this final experiment as expected (Fig. 6).

In conclusion, the developed adaptive control strategy regulates the lactic acid concentration at the target value with good performances and robustness in case of changes in temperature and setpoint. The coupling of Kalman filter and linearizing control law achieves a good tracking performance. It could be further improved by adding an integral term in the outer loop controller.

The control law leads to similar results as in the simulation study, when assuming that all the state variables are measured (Gonzalez et al. 2015a). The online estimation of the unknown dynamics helps to improve the performance in comparison to a linearizing control law coupled with a Kalman filter to estimate state variable (see e.g. (Gonzalez et al. 2015b) for the same process, and (Tebbani et al., 2015) for microalgae culture with experimental validation). The control structure is simpler and easier to implement than classical adaptive control strategies that estimate online model parameters. Indeed, since the developed control strategy is kinetic-free, the estimation algorithm is reduced to the estimation of a linear time-varying system.

\section{Conclusion}

In this paper, an adaptive control strategy is proposed to regulate the lactic acid concentration in a SSPHF continuous bioreactor. The developed controller exploits the control affine property of the model and derives a state feedback linearizing control law. This control law requires the online knowledge of all state variables (concentrations in the bulk) which are not available in the case of the studied experimental setup. Only the lactic acid concentration is determined online. Consequently, the lactic acid production rate was estimated in order to reduce the control law complexity and to make it less sensitive to model uncertainty. This production rate estimation is performed using a Kalman filter.

The obtained adaptive control law is kinetic free and simple for implementation in a real production facility. Its proof of convergence was also given (see Appendix). The control strategy was experimentally validated in a $5 \mathrm{~L}$ bioreactor operating in continuous mode. Its performance was tested for an increasing step reference profile and its robustness was studied in the case of temperature disturbance. Results proved that the control law adapts the control input variable and maintains the system at the target setpoint. Moreover, the online determination of the lactic acid production rate is performed. This represents a valuable monitoring tool of the bioprocess. The proposed control strategy uses the added sodium 
hydroxide quantity to calculate the product concentration. Consequently, there is no need of any additional sensor for the product concentration control, which reduces the costs.

Moreover, with the proposed control strategy, lactic acid production rate of $2.7 \mathrm{~g} \mathrm{~L}^{-1} \mathrm{~h}^{-1}$ was obtained when regulating the lactic acid concentration at $20 \mathrm{~g} \mathrm{~L}^{-1} \mathrm{~h}^{-1}$. This production rate value is twofold higher than the one obtained for the same process working in batch mode.

As aimed, the proposed control strategy is quite simple (no need of high computation capacity), that reduces costs (no additional sensors) and achieves a high lactic acid production (better than in batch mode). It can be used for any other lactic acid bacteria, or other operating conditions (temperature, $\mathrm{pH}$, agitation), since the control strategy is kinetic free.

This work was a preliminary study for the optimization of the industrial bio-production of lactic acid from wheat flour. Further work will consider the development of an industrial process and a future study will be conducted to test the developed control strategy at upper reactor scale and in the case of several reactors connected in series. 


\section{APPENDIX}

\section{A. Convergence analysis}

In this section, the convergence and stability of the proposed estimation and control strategies are studied.

\section{A.1 Main assumptions}

Some assumptions are made, considering the properties of the studied system. These assumptions are in fact realistic in general for microorganisms cultivated in a bioreactor.

Assumption 1. The specific growth rate $\mu$ is positive and bounded: $0 \leq \mu \leq \mu_{\max }$.

Remark 2. This assumption, realistic in the case of bacteria growth, assures also that the production rate $\gamma$ is bounded.

Assumption 2. The dilution rate is bounded $0 \leq D \leq D_{\max }$.

Remark 3. Reminding that the dilution rate is defined as the ratio between the feed flow rate and the volume and that the latter is constant, the dilution rate is then bounded. Its bounds depend on the characteristics of the used pumps.

Assumption 3. The product concentration is strictly positive and bounded $\forall D \geq 0$.

Remark 4. The state variables evolution depends on the growth rate. The latter is bounded (see Assumption 1). It depends on the substrate and production concentrations. In batch mode $(D=0)$, no nutrient is added to the culture. Consequently, the substrate will be consumed and the growth rate will decrease (since it is a limiting substrate). In addition, the product concentration will increase in the culture leading, in this study, to growth inhibition. The concentrations in the reactor remain then bounded when the reactor operates in batch mode. In continuous mode, the concentrations (substrate and product) will reach a steady-state that depends on the applied dilution rate. When the dilution is too high, the biomass concentration decreases; wash-out of the culture may occur (an equilibrium state where all biomass and product concentrations equal zero).

Assumption 4. The sampling time $T_{s}$ is chosen so that $1-D_{k} T_{s}>0$ holds $\forall k \geq 0$.

Remark 5. The sampling time can be chosen so that $1-D_{\max } T_{s}>0$. Then, from Assumption 2, Assumption 4 holds $\forall k \geq 0$. 
Assumption 5. The sampling time $T_{s}$ is as small as possible so that the discretization of the product concentration dynamics by the Euler scheme is a good approximation.

Assumption 6. The plant dynamics evolution remains bounded between two sampling instants when applying a discrete control input through a $\mathrm{ZOH}$ device.

Remark 6. This assumption is assured when the sampling time $T_{s}$ is assumed to be small and since this state variable dynamics is bounded.

Assumption 7. The error of determination of the product concentration is bounded.

Remark 7. The product concentration was determined from the sodium hydroxide addition. The error is bounded and linked to measurement errors.

Assumption 8. The matrices $\mathbf{Q}$ and $\mathbf{R}$ in the Kalman filter are positive definite. They are conservative that is $\mathbf{Q} \geq E\left(\mathbf{v}_{k} \mathbf{v}_{k}^{\mathrm{T}}\right), \mathbf{R} \geq E\left(w_{k} w_{k}^{\mathrm{T}}\right) \quad \forall k \geq 0$.

Remark 8. These matrices are design variables in the Kalman filter. They are chosen of full rank, and diagonal to simplify their tuning. The diagonal terms magnitude should be chosen to be representative of model and measurements uncertainties. For the process noise, the matrix $\mathbf{Q}$ will model the uncertainty of the model in the one hand, and the error introduced by the Euler discretization scheme in the other hand. These errors could be reduced by choosing a small sampling time $T_{s}$.

Assumption 9. The controller gain $G$ is chosen so that $0<T_{s} G<1$.

Remark 9. The lower bound of $T_{s} G$ is always fulfilled (since $T_{s}$ and $G$ are strictly positive). For the upper bound, it is restricted to the choice retained in this work. Since the sampling time is fixed from sensors characteristics, $G$ must be chosen to fulfill this assumption. It should be reminded that increasing the gain $G$ will lead to higher control input and thus to possible saturation of the control law. The choice of the gain is derived to assure a good trade-off between closed-loop time response and input saturation.

Assumption 10. The reference $P_{r e f}$ is attainable.

Remark 10. If the reference value is much higher than the initial value of $P$, the system will operate in batch mode so that the product accumulates in the reactor. However, in batch mode, the growth can decrease since the nutrients are consumed and because of the accumulation of the product (inhibition effect). The initial substrate concentration in the culture must be determined so that the reference value 
$P_{r e f}$ can be reached in the batch mode. Afterwards, the controller will switch into the continuous mode (i.e. closed-loop operating mode) to maintain the product concentration at this setpoint.

\section{A.2 Convergence of the estimation algorithm}

In this section, the convergence of the Kalman filter developed in section 3.4 is studied.

Proposition 1. The evolution matrix $\mathbf{A}_{k}$ in system (17) is invertible.

Proof. The eigenvalues of matrix $\mathbf{A}_{k}$ are 1 and $1-D_{k} T_{s}$. From Assumption 4, it comes that the latter eigenvalue is not null.

Remark 11. The inverse of matrix $\mathbf{A}_{k}$ is given by $\mathbf{A}_{k}^{-1}=\frac{1}{\beta_{k}}\left(\begin{array}{cc}1 & -T_{s} \\ 0 & \beta_{k}\end{array}\right)$ with $\beta_{k}=1-D_{k} T_{s}$.

Lemma 1. Consider the discrete-time linear system

$$
\begin{aligned}
\mathbf{z}_{k+1} & =\overline{\mathbf{A}}_{k} \mathbf{z}_{k}+\overline{\mathbf{v}}_{k} \\
\xi_{k} & =\overline{\mathbf{C}}_{k}+\bar{w}_{k}
\end{aligned}
$$

where $\overline{\mathbf{A}}_{k}$ is invertible and $\overline{\mathbf{v}}_{k} \sim \mathrm{N}(0, \overline{\mathbf{Q}})$ and $\bar{w}_{k} \sim \mathrm{N}(0, \overline{\mathbf{R}})$.

Suppose that there are positive real numbers $0<\alpha_{1}, \alpha_{2}, \sigma_{1}, \sigma_{2}<\infty$ such that the following conditions hold for some finite $K \geq 0$ and for all $k \geq K$ :

$$
\begin{aligned}
& \alpha_{1} \mathbf{I} \geq \boldsymbol{\Psi}_{1}=\sum_{i=k-K}^{k-1} \boldsymbol{\Phi}^{\mathrm{T}}(k, i+1) \overline{\mathbf{Q}} \boldsymbol{\Phi}(k, i+1) \geq \alpha_{2} \mathbf{I}, \\
& \sigma_{1} \mathbf{I} \leq \boldsymbol{\Psi}_{2}=\sum_{i=k-K}^{k} \boldsymbol{\Phi}^{\mathrm{T}}(i, k) \overline{\mathbf{C}}^{\mathrm{T}} \overline{\mathbf{R}}^{-1} \overline{\mathbf{C}} \boldsymbol{\Phi}(i, k) \leq \sigma_{2} \mathbf{I}
\end{aligned}
$$

where $\boldsymbol{\Phi}(k, i)$ is the transition matrix. For $i \leq k$, it is given by $\boldsymbol{\Phi}(k, i):=\overline{\mathbf{A}}_{k-1} \overline{\mathbf{A}}_{k-2} \cdots \overline{\mathbf{A}}_{i}, \boldsymbol{\Phi}(k, k)=\mathbf{I}$ and $\boldsymbol{\Phi}(i, k):=\boldsymbol{\Phi}(k, i)^{-1}$.

Then, $\boldsymbol{\Sigma}_{k}$, the error covariance matrix associated with the estimate using a Kalman filter is bounded $\forall k$, that is:

$$
\frac{1}{\sigma_{2}+1 / \alpha_{2}} \mathbf{I} \leq \mathbf{\Sigma}_{k} \leq\left(\alpha_{1}+1 / \sigma_{1}\right) \mathbf{I}
$$


Proof. See (Song and Grizzle, 1995). $\square$

Remark 12. Inequality $\mathbf{A} \leq \mathbf{B}$ implies that $(\mathbf{A}-\mathbf{B})$ is positive semidefinite.

Lemma 1 means that the system (A.1) is stochastically observable. Moreover, if $\overline{\mathbf{R}}=\mathbf{I}$, the pair $\left(\overline{\mathbf{A}}_{k}, \overline{\mathbf{C}}\right)$ is uniformly completely observable.

Proposition 2. The system (17) is stochastically observable and the pair $\left(\mathbf{A}_{k}, \mathbf{C}\right)$ is uniformly completely observable.

Proof. Let us consider $K=1$ and $k \geq 1$ and calculate $\boldsymbol{\Psi}_{j}, j=1,2$ in (A.2) for system (17).

In this case, the matrix $\boldsymbol{\Psi}_{1}$ is equal to $\mathbf{Q}$. From Assumption 8, Equation (A.2a) always holds from the positive definiteness of $\mathbf{Q}$.

The matrix $\boldsymbol{\Psi}_{2}$ for system (17) is given by:

$$
\boldsymbol{\Psi}_{2}=\mathbf{C}^{\mathrm{T}} \mathbf{R}^{-1} \mathbf{C}+\boldsymbol{\Phi}^{\mathrm{T}}(k-1, k) \mathbf{C}^{\mathrm{T}} \mathbf{R}^{-1} \mathbf{C} \boldsymbol{\Phi}(k-1, k)
$$

In the considered system, $\mathbf{R}$ is scalar. In addition, $\boldsymbol{\Phi}(k-1, k)=\mathbf{A}_{k-1}^{-1}$ (it exists from Proposition 1). Thus, Equation (A.3) can be further simplified:

$$
\boldsymbol{\Psi}_{2}=\frac{\mathbf{R}^{-1}}{\beta_{k-1}^{2}}\left(\begin{array}{cc}
1+\beta_{k-1}^{2} & -T_{s} \\
-T_{s} & T_{s}^{2}
\end{array}\right)
$$

with $\beta_{k-1}=1-D_{k-1} T_{s}$. From Assumptions 2 and 4, $0<1-D_{\max } T_{s} \leq \beta_{k-1} \leq 1, \forall k \geq 1$.

The matrix $\boldsymbol{\Psi}_{2}$ has two eigenvalues:

$$
\lambda_{1}=\frac{\mathbf{R}^{-1}}{\beta_{k-1}^{2}} T_{s}^{2}, \lambda_{2}=\frac{\mathbf{R}^{-1}}{\beta_{k-1}^{2}}\left(1+\beta_{k-1}^{2}\right)
$$

These two eigenvalues are strictly positive bounded reals (since $\beta_{k-1}$ is bounded and strictly positive $\forall k \geq 1$ ). Consequently, condition (A.2b) holds. The upper and lower bounds of the eigenvalues are denoted $\bar{\lambda}$ and $\underline{\lambda}$ (i.e. $\underline{\lambda} \mathbf{I} \leq \boldsymbol{\Psi}_{2} \leq \bar{\lambda} \mathbf{I}$ ).

From Lemma 1, the system is thus stochastically observable. 
Moreover, since $\mathbf{R}^{-1}$ is a scalar, $\mathbf{R}^{-1}$ can be factorized and condition (A.2b) holds for the deterministic model of the system, and thus, the pair $\left(\mathbf{A}_{k}, \mathbf{C}\right)$ is uniformly completely observable.

Proposition 3. The error covariance matrix for system (17) is bounded:

$$
\underline{p} \mathbf{I} \leq \boldsymbol{\Sigma}_{k} \leq \bar{p} \mathbf{I}
$$

with $0<p, \bar{p}<\infty$.

Proof. From Proposition 2 and Lemma 1, the error covariance matrix is bounded. The bounds $\underline{p}$ and $\bar{p}$ are function of eigenvalues of $\mathbf{Q}$ and $\boldsymbol{\Psi}_{2}$, that is from (A.2c):

$$
\underline{p}=\frac{1}{\bar{\lambda}+1 / \lambda_{\min }(\mathbf{Q})}, \bar{p}=\lambda_{\max }(\mathbf{Q})+1 / \underline{\lambda}
$$

Proposition 4. Matrix $\mathbf{A}_{k}$ and $\mathbf{C}$ of system (17) are bounded $\forall k \geq 0$, that is there are positive real numbers $\bar{a}, \bar{c}$ such that the following bounds are fulfilled $\forall k \geq 0\left\|\mathbf{A}_{k}\right\| \leq \bar{a},\|\mathbf{C}\| \leq \bar{c}$.

Remark 13. The matrix norm is defined as $\|\mathbf{A}\|=\sqrt{\lambda_{\max }\left(\mathbf{A}^{*} \mathbf{A}\right)}$, where $\lambda_{\max }$ is the largest eigenvalue and $\mathbf{A}^{*}$ is the conjugate transpose of $\mathbf{A}$.

Proof. Since the matrix $\mathbf{C}$ is constant, it is bounded (here, a possible bound is $\bar{c}=1$ ).

The Frobenius norm of $\mathbf{A}_{k}$ (defined as $\|\mathbf{A}\|_{F}=\sqrt{\sum_{i=1}^{n} \sum_{j=1}^{n}\left|a_{i j}\right|^{2}}$ ) is an upper bound of its Euclidean norm (reminding that $\beta_{k}=1-D_{k} T_{s}$ ):

$$
\left\|\mathbf{A}_{k}\right\| \leq \sqrt{1+T_{s}^{2}+\beta_{k}^{2}}
$$

Since $\beta_{k} \leq 1 \quad \forall k \geq 0\left(0<1-D_{\max } T_{s} \leq \beta_{k} \leq 1,\right),\left\|\mathbf{A}_{k}\right\|$ is then bounded $\forall k \geq 0$ :

$$
\left\|\mathbf{A}_{k}\right\| \leq \sqrt{2+T_{s}^{2}}:=\bar{a}
$$


Proposition 5. Consider the system (17) and the associated Kalman Filter. Given that the following assumptions hold:

1) There are positive numbers $\bar{a}, \bar{c}, \underline{p}, \bar{p}, \underline{q}, \underline{r}$ such that the following bounds on various matrices are fulfilled for every $k \geq 0$ :

$$
\left\|\mathbf{A}_{k}\right\| \leq \bar{a},\|\mathbf{C}\| \leq \bar{c}, \underline{p} \mathbf{I} \leq \mathbf{\Sigma}_{k} \leq \bar{p} \mathbf{I}, \underline{q} \mathbf{I} \leq \mathbf{Q}, \underline{r} \mathbf{I} \leq \mathbf{R}
$$

2) $\mathbf{A}_{k}$ is invertible $\forall k \geq 0$

then the estimation error $\tilde{\mathbf{x}}_{k}=\mathbf{x}_{k}-\hat{\mathbf{x}}_{k}$ is exponentially bounded in mean square and bounded with probability one, provided that the initial estimation error satisfies

$$
\left\|\tilde{\mathbf{x}}_{0}\right\| \leq \varepsilon
$$

and the covariance matrices of the noise terms are bounded via

$$
\mathbf{Q} \leq \delta \mathbf{I}, \mathbf{R} \leq \delta \mathbf{I}
$$

for some $\delta, \varepsilon>0$.

Proof. This proposition is derived from the Theorem 3.1 in (Reif et al., 1999). The theorem was proven in the general case of discrete-time Extended Kalman filter. Additional assumptions are needed in this theorem linked to the boundedness of the linearization residual in the dynamics (the EKF uses the jacobian matrices in the Riccati equation). Since the system (17) is bilinear, these assumptions are fulfilled (the linearization residual is null).

In condition (A.10), the boundedness of matrices $\mathbf{A}_{k}$ and $\mathbf{C}$ is fulfilled from Proposition $\mathbf{4}$, of $\boldsymbol{\Sigma}_{k}$ from Proposition 3, and of $\mathbf{Q}$ and $\mathbf{R}$ from Assumption 5.

\section{A.3 Stability of the control law}

In this section, the stability of the closed-loop system with the control law proposed in Section 3.3 is studied.

The plant dynamics is given by:

$$
\dot{P}=\gamma-D P
$$


where the control input is given by:

$D(t)=\frac{1}{\hat{P}_{k}}\left(-G\left(P_{r e f}-\hat{P}_{k}\right)+\hat{\gamma}_{k}\right):=D_{k}, \forall t \in\left[k T_{k},(k+1) T_{k}\right)$

with $\hat{P}_{k}$ is the calculated product concentration at time $t=k T_{s}$ using the measurement of the sodium hydroxide concentration, $\hat{\gamma}_{k}$ the estimated production rate and $P_{r e f}$ the reference assumed to be constant to simplify the study.

Remark 14. $\hat{P}_{k}$ could be also the estimated value with the Kalman filter.

Proposition 6. The tracking error $\tilde{P}=P-P_{\text {ref }}$ is bounded at the sampling instants $k T_{s}, \forall k>0$

Proof. The model (A.13) is discretized with an Euler scheme:

$P_{k+1}=P_{k}+T_{s}\left(\gamma_{k}-D_{k} P_{k}\right)+\delta_{k} T_{s}^{2}$

where $\delta_{k} T_{s}^{2}$ represents the discretization residual (assumed to be bounded, see Assumption 5).

From (A.14) and (A.15) it comes:

$P_{k+1}=P_{k}+T_{s}\left(\gamma_{k}-\left(\hat{\gamma}_{k}-G\left(P_{r e f}-\hat{P}_{k}\right)\right) \frac{P_{k}}{\hat{P}_{k}}\right)+\delta_{k} T_{s}^{2}$

Let us denote $\eta_{k}$ the measurement error (or the estimation error if the estimated value of $P$ is used in the control law):

$$
P_{k}=\hat{P}_{k}+\eta_{k}
$$

Then, from (A.17), (A.16) can be reformulated as:

$$
P_{k+1}=P_{k}+T_{s}\left(\gamma_{k}-\hat{\gamma}_{k}\right)+T_{s} G\left(P_{r e f}-P_{k}\right)+\left(G P_{r e f}-\hat{\gamma}_{k}\right) \frac{T_{s}}{\hat{P}_{k}} \eta_{k}+\delta_{k} T_{s}^{2}
$$

From (A.18), the dynamics of the tracking error $\tilde{P}_{k}=P_{k}-P_{r e f}$ can be deduced:

$$
\tilde{P}_{k+1}=\left(1-T_{s} G\right) \tilde{P}_{k}+T_{s}\left(\gamma_{k}-\hat{\gamma}_{k}\right)+\left(G P_{r e f}-\hat{\gamma}_{k}\right) \frac{T_{s}}{\hat{P}_{k}} \eta_{k}+\delta_{k} T_{s}^{2}
$$


From Assumption 9, the discrete-time linear system (A.19) is thus exponentially stable.

From (A.19), the error dynamics can be rewritten as a function of the error at initial time by:

$$
\tilde{P}_{k+1}=\left(1-T_{s} G\right)^{k+1} \tilde{P}_{0}+\sum_{j=0}^{k}\left(1-T_{s} G\right)^{k-j}\left(T_{s}\left(\gamma_{j}-\hat{\gamma}_{j}\right)+\left(G P_{r e f}-\hat{\gamma}_{j}\right) \frac{T_{s}}{\hat{P}_{j}} \eta_{j}+\delta_{j} T_{s}^{2}\right)
$$

It can be bounded as follows:

$$
\left|\tilde{P}_{k+1}\right| \leq\left(1-T_{s} G\right)^{k+1}\left|\tilde{P}_{0}\right|+\sum_{j=0}^{k}\left(1-T_{s} G\right)^{k-j}\left(T_{s}\left|\gamma_{j}-\hat{\gamma}_{j}\right|+\left|G P_{r e f}-\hat{\gamma}_{j}\right| \frac{T_{s}}{\hat{P}_{j}}\left|\eta_{j}\right|+\left|\delta_{j}\right| T_{s}^{2}\right)
$$

And thus:

$$
\lim _{k \rightarrow \infty}\left|\tilde{P}_{k+1}\right| \leq \lim _{k \rightarrow \infty}\left(1-T_{s} G\right)^{k+1}\left|\tilde{P}_{0}\right|+\sum_{j=0}^{k}\left(1-T_{s} G\right)^{k-j}\left(T_{s}\left|\gamma_{j}-\hat{\gamma}_{j}\right|+\left|G P_{r e f}-\hat{\gamma}_{j}\right| \frac{T_{s}}{\hat{P}_{j}}\left|\eta_{j}\right|+\left|\delta_{j}\right| T_{s}^{2}\right)
$$

From Assumption 9, this expression is simplified:

$$
\lim _{k \rightarrow \infty}\left|\tilde{P}_{k+1}\right| \leq \lim _{k \rightarrow \infty}\left[\sum_{j=0}^{k}\left(1-T_{s} G\right)^{k-j}\left(T_{s}\left|\gamma_{j}-\hat{\gamma}_{j}\right|+\left|G P_{r e f}-\hat{\gamma}_{j}\right| \frac{T_{s}}{\hat{P}_{j}}\left|\eta_{j}\right|+\left|\delta_{j}\right| T_{s}^{2}\right)\right]
$$

Since the estimation error is bounded (Proposition 5), and from Assumptions 1, 3, 5 and 7, it comes:

$$
\forall j \quad\left|\gamma_{j}-\hat{\gamma}_{j}\right| \leq \tilde{\gamma}_{\max }, \frac{\left|G P_{r e f}-\hat{\gamma}_{j}\right|}{\hat{P}_{j}} \leq \rho_{\max },\left|\eta_{j}\right| \leq \eta_{\max },\left|\delta_{j}\right| \leq \delta_{\max }
$$

with $\tilde{\gamma}_{\max }, \rho_{\max }, \eta_{\max }, \delta_{\max }$ real positive, denoting the maximum values of the associated expressions.

Consequently, the error in (A.23) can be bounded by:

$$
\begin{aligned}
\lim _{k \rightarrow \infty}\left|\tilde{P}_{k+1}\right| & \leq\left(T_{s} \tilde{\gamma}_{\max }+T_{s} \rho_{\max } \eta_{\max }+\delta_{\max } T_{s}^{2}\right) \lim _{k \rightarrow \infty} \sum_{j=0}^{k}\left(1-T_{s} G\right)^{k-j} \\
& \leq\left(\tilde{\gamma}_{\max }+\rho_{\max } \eta_{\max }+\delta_{\max } T_{s}\right) \frac{1}{G}
\end{aligned}
$$

The tracking error is then bounded at the sampling instants $k T_{S} \forall k \geq 0$, and can be reduced by an appropriate choice of the gain $G$. 
Remark 15. Without any estimation, measurement and modeling errors, the error tends to zero.

Proposition 7. The tracking error $\tilde{P}(t)=P(t)-P_{r e f}$ is bounded $\forall t \geq 0$ and can be adjusted by an appropriate $G$ gain value.

Proof. From Proposition 6, Assumption 6 and (A.25), the Proposition 7 can be deduced. $\square$ 


\section{References}

Abdel-Rahman M, Tashiro Y. \& Sonomoto K. (2013) Recent advances in lactic acid production by microbial fermentation processes. Biotechnology Advances, 31: 877-902.

Bastin G \& Dochain D (1990). Online estimation and adaptive control of bioreactors, Process measurement and Control. Elsevier, Amsterdam.

Ben Youssef C, Guillou V, Olmos-Dichara A (2000) Modelling and adaptive control strategy in a lactic fermentation process. Control Eng Pract. 8: 1297-1307. DOI:10.1016/S0967-0661(00)00061-7.

Ben Youssef C, Goma G \& Olmos-Dichara A (2005) Kinectic modelling of lactobacillus casei ssp. rhamnosus growth and lactic acid production in batch cultures under various medium conditions. Biotechnol Lett. 27:1785-1789.DOI: 10.1016/S0967-0661(00)00061-7.

Choi M, Al-Zahrani S M, Lee S Y (2014) Kinetic model-based feed-forward controlled fed-batch fermentation of Lactobacillus rhamnosus for the production of lactic acid from Arabic data juice. Bioproc Biosyst Eng. 37: 1007-1015. DOI 10.1007/s00449-013-1071-7.

Dahhou B, Chamilothoris G, Roux G (1991) Adaptive predictive control of a continuous fermentation process. Int J Adapt Control. 5: 351-362.DOI: 10.1002/acs.4480050602.

Dai W, Word D P, Hahn J (2014). Modeling and dynamic optimization of fuel-grade ethanol fermentation using fed-batch process. Control Eng Pract. 22:231-241.

Friedman M (2004) Applications of the ninhydrin reaction for analysis of amino acids, peptides and proteins to agricultural and biomedical sciences. J Agric Food Chem. 52:385-406. DOI:10.1021/jf030490p.

Gonzalez K, Tebbani S, Dumur D, Lopes F, Pareau D, Givry S, Thorigné A (2015a) Adaptive control of lactic acid production process from wheat flour. In Proceedings of 9th IFAC Symposium on Advanced Control of Chemical Processes ADCHEM 2015, June 2015, Whistler, Canada. DOI: 10.1016/j.ifacol.2015.09.113.

Gonzalez K, Tebbani S, Dumur D, Lopes F, Pareau D, Givry S, Thorigné A (2015b) Feedback linearizing controller coupled to an Unscented Kalman filter for lactic acid regulation. In Proceedings of 19th International Conference on System Theory, Control and Computing (ICSTCC), Oct 2015, Cheile Gradistei, Romania. DOI: 10.1109/ICSTCC.2015.7321296. 
Gonzalez K, Tebbani S, Lopes F, Givry S, Thorigné A, Dumur D, Pareau D (2016) Modelling the continuous lactic acid production process from wheat flour. Appl. Microbiol. Biotechnol., 100:147159. DOI: $10.1007 / \mathrm{s} 00253-015-6949-7$.

Hilgert N, Harmand J, Steyer J P, Vila J P (2004) Non parametric identification and adaptive control of an anaerobic fluidized bed digester. Control Eng Pract. 8: 264-272. DOI:10.1016/S09670661(99)00165-3.

Hofvendahl K, Hahn-Hägerdal B (2000) Factors affecting the fermentative lactic acid production from renewable resources. Enzym. Microb. Tech. 26:87-107. doi: 10.1016/S0141-0229(99)00155-6.

Isidori A (1989) Nonlinear control systems, Berlin, Springer-Verlag.

Jenzch M, Simutis R, Luebbert A (2006) Generic model control of the specific growth rate in recombinant Escherichia coli cultivations. J Biotechnol. 122: 483-493. DOI: 10.1016/j.jbiotec.2005.09.013.

Kwon S, Yoo I K, Lee W G, Chang H N, Chang Y K (2001) High-rate continuous production of lactic acid by lactobacillus rhamnosus in a two-stage membrane cell-recycle bioreactor, Biotechnol Bioeng. 73: 25-34. DOI: 10.1002/1097-0290(20010405)73:1<25::AID-BIT1034>3.0.CO;2-N.

Levenspiel O (1980) The Monod equation: a revisit and generalization to product inhibition situation. Biotechnol. Bioeng., 22:1671-1687. doi: 10.1002/bit.260220810.

Lewis F L, Xie L, Popa D (2008) Optimal and Robust Estimation: With an Introduction to Stochastic Control Theory, Automation and control engineering, vol 26, 2nd ed. FL : CRS Press, Boca Raton.

Mailleret L, Bernard O, Steyer J P (2004) Nonlinear adaptive control for bioreactor with unknown kinetics. Automatica. 40: 1397-1385. DOI: 10.1016/j.automatica.2004.01.030.

Marcos N I, Guay M, Dochain D (2004) Output feedback adaptive extremum-seeking control of a continuous stirred tank bioreactor with Monod's kinetics. J Process Contr., 14: 807-818. DOI: 10.1016/j.jprocont.2003.12.002.

Niemelä S, Keskus M (2002) Uncertainty of quantitative determinations derived by cultivation of microorganisms. Centre for Metrology and Accreditation, Finland.

Ochoa S, Lyubenova V, Repke J U, Ignatova M, Wozny G (2008) Adaptive control of the simultaneous saccharification-fermentation process from starch to ethanol. Computer Aided Chemical Engineering, 25:489-494.DOI:10.1016/S1570-7946(08)80086-7. 
Pons M N (1991) Bioprocess monitoring and control. Munich: Hanser Publishers.

Petre E, Selişteanu D, Şendrescu D (2011) Neural networks based adaptive control of a fermentation bioprocess for lactic acid production. Proceedings of the 3rd International Conference on Intelligent Decision Technologies, SIST 10, 201-212. DOI: 10.1007/978-3-642-22194-1_21.

Ramaswamy S, Cutright T J, Qammar H K (2005) Control of a continuous bioreactor using model predictive control. Process Biochem. 40: 2763-2770. DOI:10.1016/j.procbio.2004.12.019.

Reif K., Gunther S., Yaz E., Unbehauen R., (1999) Stochastic stability of the discrete-time extended Kalman filter, IEEE Tansactions on Automatic Control, 44:714-728.

Roux G, Dahhou B, Queinnec I (1996) Modelling and estimation aspects of adaptive predictive control in a fermentation process. Control Eng Pract., 4: 55-66. DOI: 10.1016/0307-904X(92)90004-M.

Schubert J, Simutis R, Dors M, Havlik I, Lübbert, A (1994) Bioprocess optimization and control : Application of hybrid modelling. J Biotechnol., 35: 51-68. DOI: 10.1016/0168-1656(94)90189-9.

Sbarciog M, Coutinho D, Vande Wouwer A (2014) A simple output-feedback strategy for the control of perfused mammalian cell cultures, Control Eng. Pract., 32:123-135.

Selișteanu D, Petre E, Răsvan V B (2007) Sliding mode and adaptive sliding-mode control of a class of nonlinear bioprocesses. Int J Adapt Control Signal Process. 21:795-822. DOI: 10.1002/acs.973.

Shi Z, Shimizu K, Iijima S, Morisue T, Kobayashi T (1990) Adaptive on-line optimizing control for lactic acid fermentation. J Ferment bioeng., 70:415-419. DOI: 10.1016/0922-338X(90)90124-F.

Song Y, Grizzle J W (1995) The extended Kalman filter as a local asymptotic observer for discrete-time nonlinear systems, Journal of Mathematical Systems, Estimation and Control, 5:59-78.

Tebbani S, Lopes F, Becerra Celis G (2015) Nonlinear control of continuous cultures of Porphyridium purpureum in a photobioreactor. Chemical Engineering Science, 123:207-219. 


\section{Tables}

Table 1. Model parameters

\begin{tabular}{ll}
\hline Parameter & Value \\
\hline$\mu_{\max }\left(\mathrm{h}^{-1}\right)$ & 0.28 \\
$P_{\max }\left(\mathrm{g} \mathrm{L}^{-1}\right)$ & 98.6 \\
$Y_{X S}\left(\mathrm{~g} \mathrm{~g}^{-1}\right)$ & 0.053 \\
$Y_{P S}\left(\mathrm{~g} \mathrm{~g}^{-1}\right)$ & 0.82 \\
$K_{M}\left(\mathrm{~h}^{-1}\right)$ & 0.035 \\
$K_{S}\left(\mathrm{~g} \mathrm{~L}^{-1}\right)$ & 0.5 \\
$n$ & 3 \\
\hline
\end{tabular}




\section{Figures}

Fig. 1 Scheme of the 5L bioreactor used for the SSPHF step. In red the offline measurements, in blue the controlled variables and in black the feeding and output flows.

Fig. 2 Bioreactors configuration to perform the continuous SSPHF. $X, S, M$ and $P$, biomass, glucose, maltose and lactic acid concentrations, respectively. $M_{0}$ and $S_{0}$, maltose and glucose concentrations feeding the bioreactor, respectively.

Fig. 3 Feedback linearizing control architecture.

Fig. 4 Implemented adaptive Feedback linearizing control.

Fig. 5 Adaptive control law experimental validation. A Lactic acid concentration evolution with time. $P_{r e f 1}=20 \mathrm{~g} / \mathrm{L}$ (dashed line), $P_{r e f 2}=27 \mathrm{~g} / \mathrm{L}$ (dotted line), online calculated lactic acid concentration ( solid line), offline calculated lactic acid concentration $(\bullet)$. B Dilution rate evolution with time. C Estimated production rate. D Offline measurements of cells $* 25(\bullet)$, glucose $(\mathbf{\Delta})$ and maltose $(\boldsymbol{\square})$ concentrations.

Fig.6 Adaptive control law robustness study. A Temperature disturbance. B Lactic acid concentration evolution with time. $P_{r e f}=27 \mathrm{~g} / \mathrm{L}$ (dotted line), online calculated lactic acid (solid line) and offline measurement of lactic acid concentration ( $\bullet$ ). C Dilution rate evolution with time. D Estimated production rate. E Offline measurements of cells*25 $(\bullet)$, glucose $(\boldsymbol{\Delta})$ and maltose $(\boldsymbol{\square})$ concentrations. 


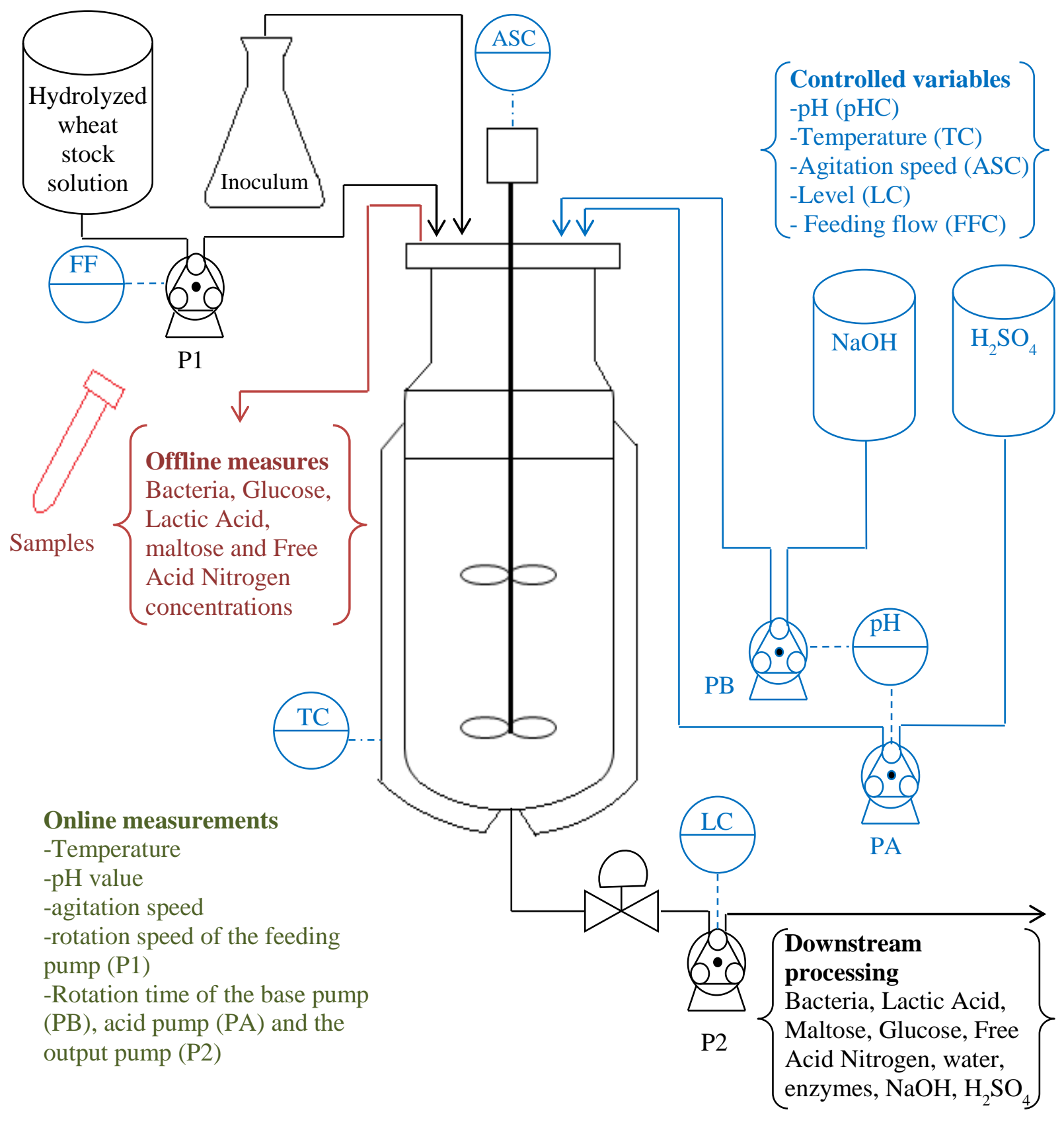

Fig. 1 
Hydrolyzed

wheat stock

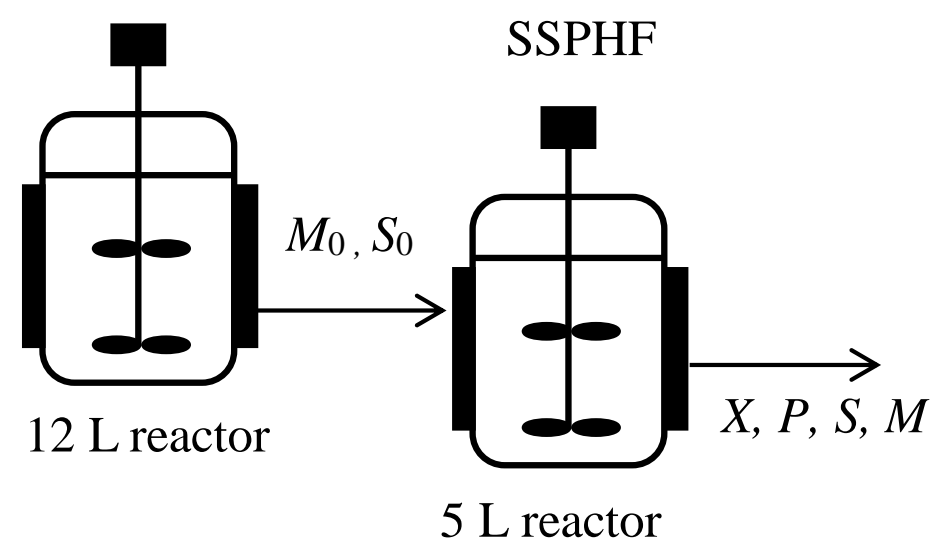

Fig. 2 


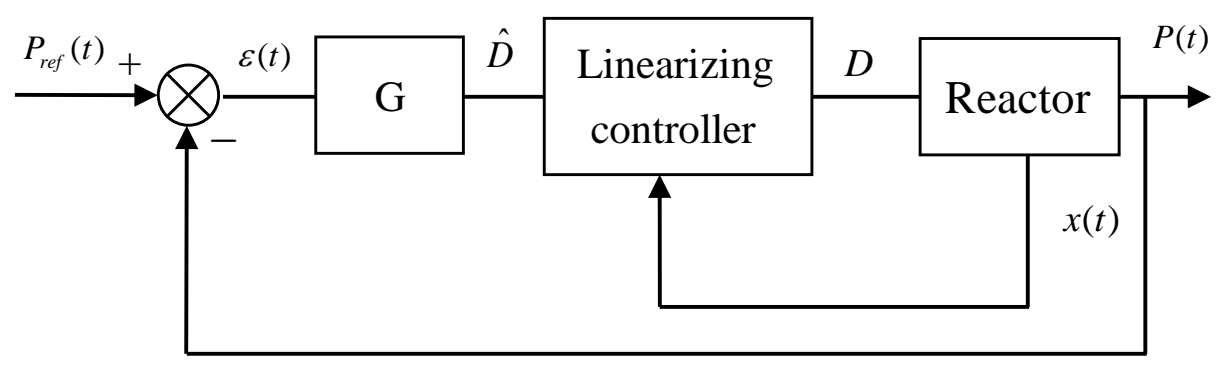

Fig. 3 


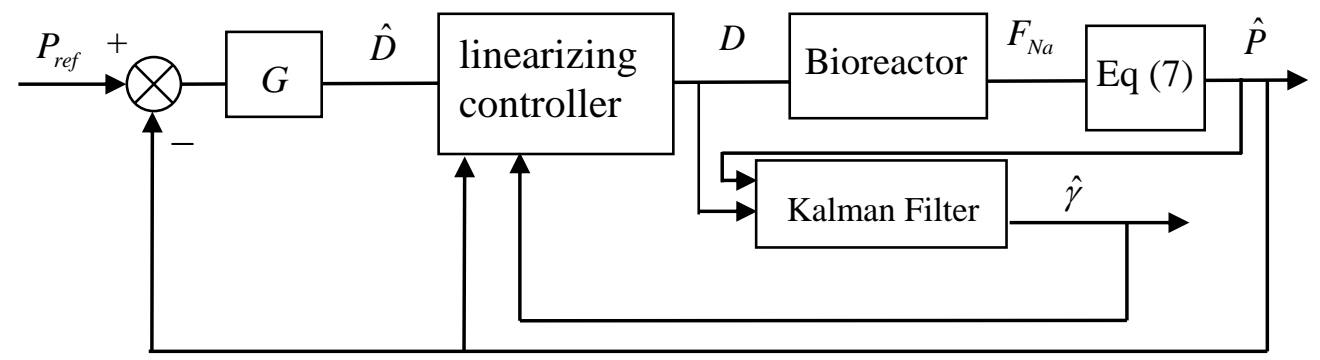

Fig. 4 


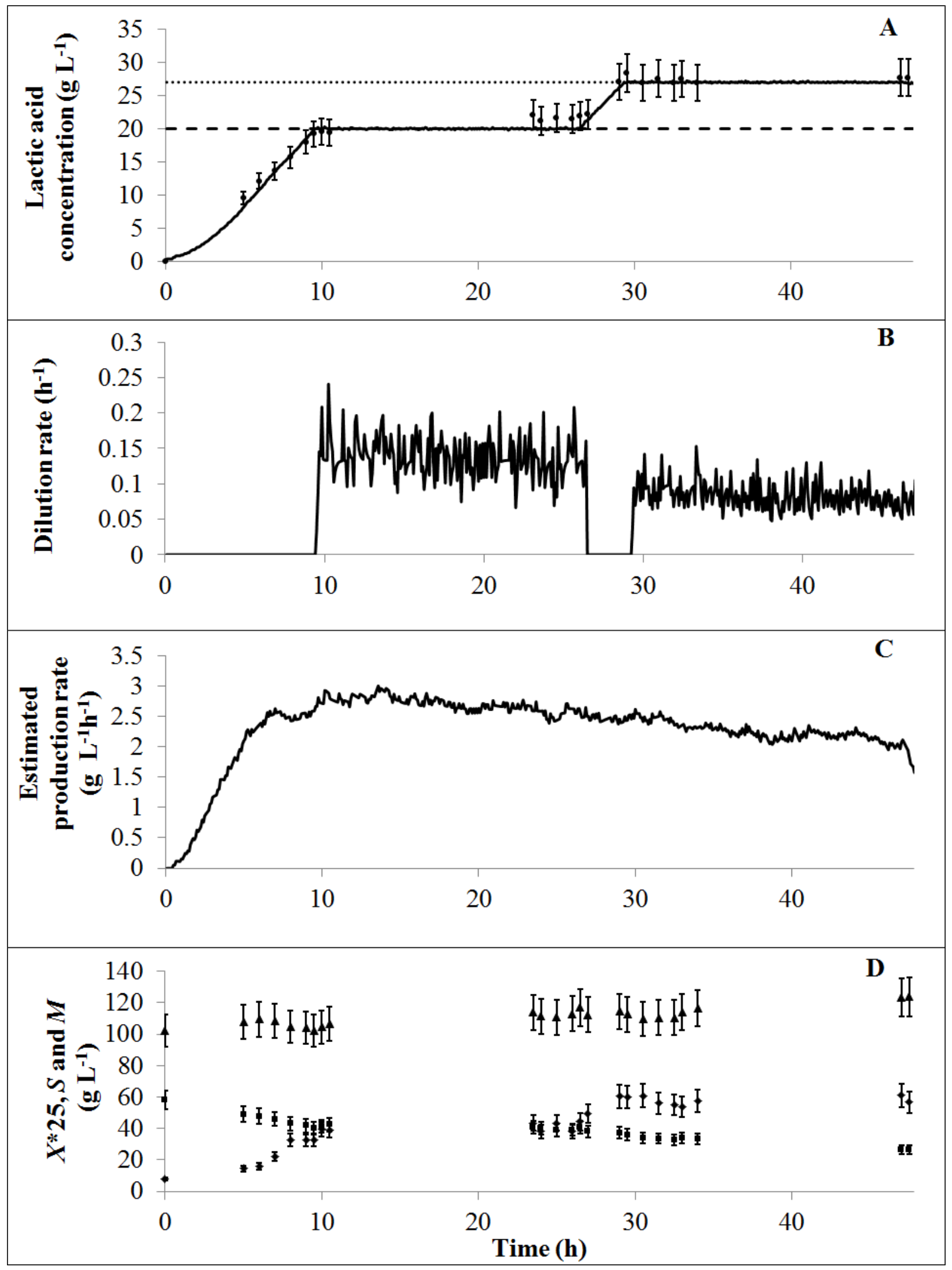

Fig. 5 


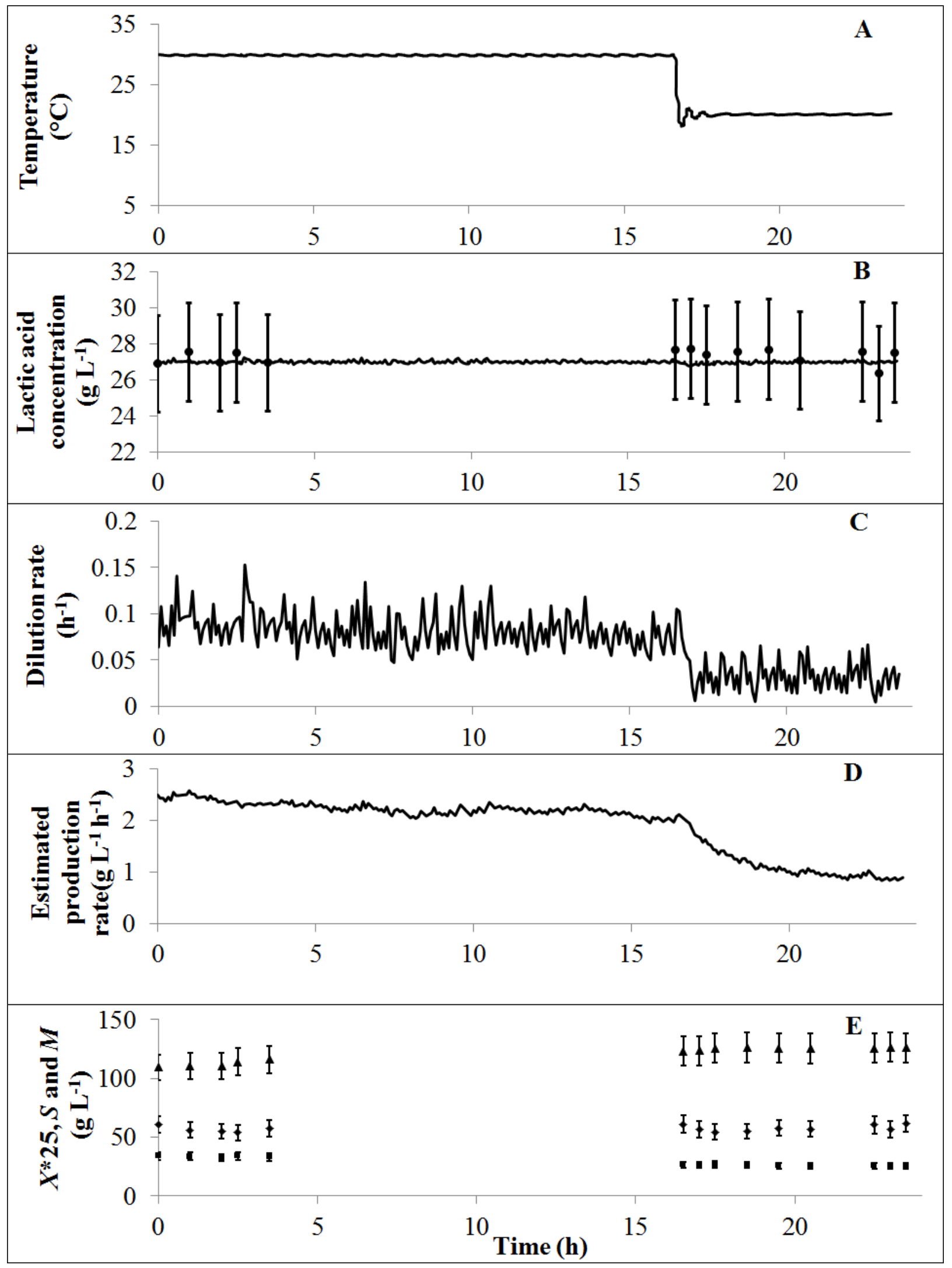

Fig. 6 